\title{
Audit of extracapsular cataract extraction and posterior chamber lens implantation as a routine treatment for age related cataract in east Africa
}

\author{
David Yorston, Allen Foster
}

\begin{abstract}
Aims-To evaluate the outcome of extracapsular cataract extraction (ECCE) and posterior chamber intraocular lens implantation (PC-IOL) in an African eye clinic during the transition from intracapsular cataract extraction to ECCE and PC-IOL.

Methods-A retrospective survey of 461 consecutive operations for age related cataract with a mean follow up of 52.9 weeks (range 0-275) and a minimum follow up of 4 weeks in $87.9 \%$ of eyes. Results-A best corrected vision of $6 / 18$ or better was obtained in $94.3 \%$ of eyes, and an uncorrected vision of $6 / 18$ or better in $78.2 \%$ of eyes. Six eyes $(1.5 \%)$ had a best corrected vision of less than 6/60. The visual acuity at 2 months was strongly predictive of the vision at 1 year or more after surgery. Preoperative biometry and IOL power calculation increased the proportion of eyes obtaining an uncorrected vision of $6 / 18$ or better from $73.8 \%$ to $\mathbf{8 1 . 3 \%}$. Four eyes developed visually significant posterior capsule opacity.

Conclusion-ECCE and PC-IOL can give very good results in an African setting. A better visual outcome should lead to increased demand for cataract surgery, which will eventually reduce the number of cataract blind people in Africa.

(Br F Ophthalmol 1999;83:897-901)
\end{abstract}

It is estimated that about 3.5 million people are blind from cataract in sub-Saharan Africa. As the population grows, and ages, this figure is likely to increase.

Most of the cataract operations carried out in Africa are intracapsular extractions (ICCE), with provision of standard glasses for aphakic correction. However, spectacles are not the ideal treatment for aphakia, particularly in a population that may lose or break their glasses, and may be unable to afford to replace them.

Good results are obtained with extracapsular cataract extraction (ECCE), and posterior chamber lens implants (PC-IOL) in the industrialised countries. The purpose of this study was to audit the results obtained by a single surgeon, making the transition from ICCE to ECCE and PC-IOL as the routine treatment of age related cataract.

Dr Allen Foster, London School of Hygiene and

Tropical Medicine, Keppel

Street, London WC1E 7HT.

Accepted for publication 22 March 1999

Methods

All patients were operated on by one of the authors (DY). Before 1992, ICCE with specta- cle correction was the routine treatment for age related cataract. From 1992, ECCE and PC-IOL was the operation of choice for all patients with cataract treated by DY at Kikuyu Eye Unit, a large eye clinic $20 \mathrm{~km}$ from the centre of Nairobi.

The notes of 461 consecutive patients, operated between 1 January 1992 and 31 December 1995, who were aged 50 or over at the time of surgery, were reviewed. Patients who had not attended the clinic for over a year were contacted by letter at their last known address.

Preoperatively all patients received at least four doses of topical chloramphenicol or gentamicin eye drops, and two doses of 5\% povidone iodine eye drops immediately before surgery.

Biometry was carried out routinely during the last 9 months of the 4 year study period, and the lens power was calculated using the SRK-II formula. Before that date, patients routinely received a $20-22 \mathrm{D}$ implant, depending on availability. This was modified if information was available about the preoperative refractive state of the eye, or about the refractive state of the fellow eye following IOL surgery.

A posterior limbal section was used in almost all cases. Every effort was made to ensure symmetrical placement of the IOL in the capsular bag. In immature cataracts, a continuous curvilinear capsulorhexis (CCC), with hydroexpression of the nucleus was performed. In mature lenses, a linear capsulotomy was performed between 10 o'clock and 2 o'clock; following removal of the nucleus and soft lens matter, the IOL was inserted, and the central anterior capsule was removed with capsulorhexis forceps. The section was closed with three or five interrupted $9 / 0$ nylon sutures. Viscoelastic was used for IOL insertion only.

Postoperatively all patients received prednisolone sodium phosphate $1 \%$ for at least 6 weeks. This was supplemented, if necessary, by subconjunctival dexamethasone, or systemic prednisolone.

Patients were usually discharged on the second postoperative day, and advised to return for outpatient follow up 1 week after surgery. They were seen again, and refracted, 2 months after surgery. Some patients did not return for follow up, and, of those who did, patients with good uncorrected vision were often not refracted.

Any patient with a vision of less than 6/18, 
Table 1 Operative complications in 461 ECCE with PC-IOL procedures, by type of capsulectomy, Kikuyu hospital

\begin{tabular}{|c|c|c|c|c|c|c|}
\hline \multirow{2}{*}{$\frac{\text { Complication }}{\text { Nil }}$} & \multicolumn{2}{|c|}{ Capsulorhexis } & \multicolumn{2}{|c|}{$\begin{array}{l}\text { Linear } \\
\text { capsulotomy }\end{array}$} & \multicolumn{2}{|c|}{ Total } \\
\hline & 223 & $89.6 \%$ & 196 & $92.5 \%$ & 419 & $90.9 \%$ \\
\hline PC rupture, no vitreous loss & 9 & $3.6 \%$ & 10 & $4.7 \%$ & 19 & $4.1 \%$ \\
\hline $\mathrm{PC}$ rupture and vitreous loss & 13 & $5.2 \%$ & 1 & $0.5 \%$ & 14 & $3.0 \%$ \\
\hline Zonular dehiscence, no vitreous loss & 1 & $0.4 \%$ & 2 & $0.9 \%$ & 3 & $0.7 \%$ \\
\hline Zonular dehiscence and vitreous loss & 3 & $1.2 \%$ & 2 & $0.9 \%$ & 5 & $1.1 \%$ \\
\hline Iridodialysis & 0 & $0.0 \%$ & 1 & $0.5 \%$ & 1 & $0.2 \%$ \\
\hline Total & 249 & $100 \%$ & 212 & $100 \%$ & 461 & $100 \%$ \\
\hline
\end{tabular}

Table 2 Powers of IOL used in 461 ECCE procedures at Kikuyu hospital

\begin{tabular}{llllll}
\hline & \multicolumn{2}{l}{ No biometry } & & \multicolumn{2}{l}{ Biometry $^{*}$} \\
\cline { 2 - 3 } \cline { 5 - 5 } Average power & 20.87 & & & 21.79 \\
\hline$<20 \mathrm{D}$ & 23 & $6.3 \%$ & & 10 & $10 \%$ \\
$20-22 \mathrm{D}$ & 327 & $90.6 \%$ & & 57 & $57 \%$ \\
$>22 \mathrm{D}$ & 11 & $3.1 \%$ & & 33 & $33 \%$ \\
Total & 361 & $100 \%$ & & 100 & $100 \%$ \\
\hline
\end{tabular}

$\chi^{2}=85.7 \mathrm{p}<0.001 .{ }^{\star} \mathrm{A}$-constant $=118.5$.

\section{Results}

PATIENTS

A total of 461 eyes in 411 patients were included in the study. The average age of the patients at operation was 61.3 years (SD 9.1). There were 159 (38.7\%) women. Eighty five per cent of the patients were of African ethnic origin. The remainder were east African Asians.

Of 461 operated eyes, follow up of at least 4 weeks was available on 404 (87.6\%). In all, 346 eyes $(75.1 \%)$ were examined at approximately 2 months after surgery. A total of 184 eyes $(39.9 \%)$ were followed for at least 1 year, 95 $(20.6 \%)$ for at least 2 years, and $36(7.8 \%)$ for at least 3 years. The mean follow up for all 461 eyes was 55.9 weeks (range $0-275$ ).

There were no significant differences in age, sex, preoperative visual acuity, type of surgery performed, or intraoperative complications between the 405 eyes with 4 weeks or more of follow up, and the 56 eyes with less than 4 weeks of follow up.

\section{SURGERY AND COMPLICATIONS}

A linear capsulotomy was performed in 212 eyes, and CCC in 246 eyes. Three eyes had an attempted capsulorhexis that was completed by a can opener capsulotomy.

Out of 461 operations, $90.9 \%$ were completed without significant intraoperative complications (see Table 1). The most frequent complication was posterior capsule rupture, which occurred in 33 eyes $(7.2 \%)$. In most cases it was possible to insert an IOL into the sulcus following capsule rupture, but in three eyes there was insufficient capsule remaining,

Table 3 Preoperative visual acuity in 461 eyes having ECCE and PC-IOL, and visual outcome in 404 eyes seen at least 4 weeks after surgery, uncorrected and with full correction

\begin{tabular}{|c|c|c|c|c|c|c|}
\hline & \multicolumn{2}{|c|}{ Preoperative acuity } & \multicolumn{2}{|c|}{$\begin{array}{l}\text { Latest uncorrected } \\
\text { acuity }\end{array}$} & \multicolumn{2}{|c|}{ Latest corrected acuity } \\
\hline & $n$ & $\%$ & $n$ & $\%$ & $n$ & $\%$ \\
\hline $6 / 4-6 / 18$ & 0 & 0 & 316 & 78.2 & 381 & 94.3 \\
\hline$<6 / 18-6 / 60$ & 43 & 9.3 & 79 & 19.6 & 17 & 4.2 \\
\hline$<6 / 60-3 / 60$ & 28 & 6.1 & 3 & 0.7 & 0 & 0 \\
\hline$<3 / 60$ & 390 & 84.6 & 6 & 1.5 & 6 & 1.5 \\
\hline Total & 461 & 100 & 404 & 100 & 404 & 100 \\
\hline
\end{tabular}

Table 4 Visual acuities at 2 months and 1 year in 140 eyes having ECCE with PC-IOL at Kikuyu hospital

\begin{tabular}{l|lll}
\hline & $\begin{array}{l}\text { One year } \\
6 / 18+\end{array}$ & $<6 / 18$ & Total \\
\hline $\begin{array}{l}\text { Two months } \\
6 / 18+\end{array}$ & & & \\
$<6 / 18$ & 126 & 2 & 128 \\
Total & 10 & 2 & 12 \\
\hline
\end{tabular}

and a flexible, open loop anterior chamber IOL was inserted instead. Vitreous loss was encountered in 19 cases $(4.6 \%)$ of which three occurred following linear capsulotomy $(1.4 \%)$, 11 during the first 100 CCC (11\%), and four during the next 146 CCC procedures $(2.7 \%)$. One episode of vitreous loss occurred following a can opener capsulotomy. Out of the 404 patients with at least 4 weeks' follow up, 35 had an intraoperative complication, of whom four $(11.4 \%)$ had a latest corrected vision of less than $6 / 18$. In those with no surgical complications, $19(5.2 \%)$ had a latest corrected vision of less than $6 / 18\left(\chi^{2}=2.35, \mathrm{p}>0.05\right)$.

All 458 PC-IOLs were biconvex. Of these, $363(79.3 \%)$ were single piece PMMA lenses, and $95(20.7 \%)$ were three piece PMMA lenses. The powers of lens used varied according to whether or not biometry was available (see Table 2).

\section{VISUAL RESULTS}

The preoperative and latest corrected and uncorrected visions are shown in Table 3. Postoperative visions are only given for the 404 eyes with a follow up of at least 4 weeks.

Preoperatively $390(84.6 \%)$ of eyes were blind (best corrected acuity <3/60). Postoperatively, six eyes $(1.5 \%)$ were blind. A latest corrected vision of $6 / 18$ or better was obtained in $94.3 \%$ of eyes. An uncorrected vision of $6 / 18$ or better was obtained in $78.2 \%$ of eyes at their last follow up visit. There was no difference in visual outcome between CCC and linear capsulotomy, with $94.7 \%$ and $94.2 \%$ respectively, achieving $6 / 18$ or better.

The visual acuity at 2 months in 346 eyes was compared with the visual acuity at 1 year for the 140 eyes for which paired data were available. The results are shown in Table 4 . Two eyes $(1.4 \%)$ with an acuity of $6 / 18$ or better at 2 months deteriorated by 1 year, and 10 eyes $(7.1 \%)$ with an acuity of less than $6 / 18$ at 2 months improved to $6 / 18$ or better by 1 year. The positive predictive value of a visual acuity of $6 / 18$ or better at 2 months for a vision of $6 / 18$ a year or more later was $98.4 \%$. A vision of less than $6 / 18$ at 2 months was less reliably predictive of a poor outcome at 1 year.

Six patients had a poor visual outcome (best corrected vision less than 6/60). The causes of poor vision are given in Table 5.

\section{BIOMETRY AND REFRACTIVE OUTCOME}

Out of 404 eyes with follow up data, refractions were performed on $330(81.6 \%)$. A total of 66 $(20.0 \%)$ patients had an absolute spherical error greater than or equal to 2 dioptres (Table 6).

An absolute spherical error exceeding 2 dioptres was significantly more likely in pa- 
Table 5 Cause of poor vision (latest corrected VA <6/60) in six eyes following 461 ECCE with PC-IOL procedures at Kikuyu hospital

\begin{tabular}{ll}
\hline Latest VA & Complications \\
\hline $2 / 60$ & Pre-existing retinal detachment. Reattached with scleral buckle \\
$2 / 60$ & $\begin{array}{l}\text { Pre-existing retinal detachment and macular hole. Reattached with vitrectomy, oil, } \\
\text { and buckle }\end{array}$ \\
$2 / 60$ & Post capsule opacity, refused YAG laser \\
HM & Retinitis pigmentosa \\
PL & Proliferative diabetic retinopathy, rubeotic glaucoma \\
NPL & Suppurative keratitis 9 weeks postop. Endophthalmitis and evisceration \\
\hline
\end{tabular}

Table 6 Results of refraction in 330 eyes undergoing ECCE and PC-IOL, by availability of biometry

\begin{tabular}{|c|c|c|c|c|}
\hline & \multicolumn{2}{|c|}{ No biometry } & \multicolumn{2}{|c|}{ Biometry } \\
\hline & $n$ & $\%$ & $n$ & $\%$ \\
\hline \multicolumn{5}{|c|}{ Absolute spherical error: } \\
\hline $0-0.99$ & 117 & 45.2 & 38 & 53.5 \\
\hline $1.0-1.99$ & 83 & 32.0 & 26 & 36.6 \\
\hline $2.0-2.99$ & 43 & 16.6 & 5 & 7.0 \\
\hline $3.0-3.99$ & 13 & 5.0 & 1 & 1.4 \\
\hline $4.0+$ & 3 & 1.2 & 1 & 1.4 \\
\hline Total & 259 & 100.0 & 71 & 100.0 \\
\hline \multicolumn{5}{|l|}{ Cylinder: } \\
\hline $0-0.99$ & 123 & 47.5 & 24 & 33.8 \\
\hline $1.0-1.99$ & 79 & 30.5 & 22 & 31.0 \\
\hline $2.0-2.99$ & 46 & 17.8 & 20 & 28.2 \\
\hline $3.0-3.99$ & 3 & 1.2 & 4 & 5.6 \\
\hline $4.0+$ & 8 & 3.1 & 1 & 1.4 \\
\hline Total & 259 & 100.0 & 71 & 100.0 \\
\hline \multicolumn{5}{|c|}{ Uncorrected VA: } \\
\hline $6 / 4-6 / 6$ & 55 & 17.3 & 11 & 12.8 \\
\hline $6 / 9$ & 88 & 27.7 & 20 & 23.3 \\
\hline $6 / 12$ & 48 & 15.1 & 17 & 19.8 \\
\hline $6 / 18$ & 56 & 17.6 & 19 & 22.1 \\
\hline$<6 / 18$ & 71 & 22.3 & 19 & 22.1 \\
\hline Total & 318 & 100.0 & 86 & 100.0 \\
\hline
\end{tabular}

tients who had not had biometry $\left(\chi^{2}=4.3\right.$, $0.05>p>0.01)$. Following biometry, the IOL was chosen with the intention of making the patient emmetropic. In practice, biometry was associated with slight myopia. The mean spherical error in patients who had biometry was $-0.83 \mathrm{D}$, and in patients who did not have biometry, the mean sphere was -0.18 (diff $=$ $-0.65,95 \% \mathrm{CI}=-0.99$ to -0.31$)$.

Although biometry reduced the number of patients with large spherical errors, it did not have a significant impact on the numbers of patients achieving unaided visions of $6 / 18$ or better (see Table 7).

The average astigmatism at 2 months was $1.53 \mathrm{D}$ (SD 1.58). Fifty four (15.6\%) eyes had more than 2 dioptres of astigmatism at 2 months after surgery. Nine patients who had at least 4 dioptres of "with the rule" astigmatism underwent selective suture removal.

POSTOPERATIVE COMPLICATIONS

The most frequent complication was severe uveitis (Table 8). This was defined as uveitis with obvious fibrin formation in the anterior chamber. The uveitis did not persist beyond 4 weeks postoperatively. No cases of endoph-

Table 7 Uncorrected visual acuities at 2 months with, and without, biometry in the 346 eyes that were examined approximately 2 months after surgery

\begin{tabular}{llll}
\hline & No of eyes & $\begin{array}{l}\text { Uncorrected } \\
\text { VA }<6 / 18\end{array}$ & $\begin{array}{l}\text { Uncorrected } \\
\text { VA 6/18+ }\end{array}$ \\
\hline Biometry & 74 & $16(21.6 \%)$ & $58(78.3 \%)$ \\
No biometry & 272 & $77(28.3 \%)$ & $195(71.7 \%)$ \\
Total & 346 & $93(26.9 \%)$ & $253(73.1 \%)$ \\
\hline$\chi^{2}=1.32, p>0.2$ & & &
\end{tabular}

$\chi^{2}=1.32, \mathrm{p}>0.2$.
Table 8 Postoperative complications following 461 ECCE with PC-IOL procedures at Kikuyu hospital

\begin{tabular}{ll}
\hline No of eyes & Complication \\
\hline & Early \\
15 & Severe uveitis postop \\
9 & Hyphaema \\
4 & Raised IOP postop \\
1 & Retained soft lens matter \\
& Late \\
13 & Diabetic retinopathy \\
10 & Sutures removed for astigmatism \\
5 & Cystoid macular oedema \\
4 & Post capsule opacity \\
2 & YAG to anterior capsule remnant \\
1 & Developed macular hole 2 years postop \\
1 & Suppurative keratitis 9 weeks postop. \\
1 & Endophthalmitis and evisceration \\
\hline
\end{tabular}

thalmitis, iris prolapse, or corneal decompensation were seen.

Visually significant posterior capsule opacity was uncommon. No patients had a planned primary posterior capsulotomy. Posterior capsulotomy was performed if the vision was less than $6 / 18$, and there was visible capsular opacity.

Three patients had a YAG posterior capsulotomy. One patient was diagnosed as having significant capsule opacity, but refused treatment. Out of 95 eyes followed for a minimum of 2 years, four $(4.2 \%)$ required posterior capsulotomy. In these four patients, posterior capsule opacification was noted at $67,107,141$, and 161 weeks after surgery.

\section{Discussion}

PATIENTS

Poor follow up is a problem in Africa. Patients may be forced to travel long distances in order to obtain treatment. This study has a minimum of 1 month's follow up on $87.6 \%$ of eyes, and 2 months' follow up on more than three quarters of them. This is comparable with other similar studies. ${ }^{2} 3$

\section{SURGERY}

The aim of surgery was to minimise the risk of capsular opacity by removing all the lens matter, and placing a biconvex lens in the capsular bag. ${ }^{4}$ In mature and hypermature cataracts capsulorhexis is difficult, as liquid lens matter obscures the anterior capsule as soon as the lens is punctured. In these cases a linear capsulotomy enables the lens to be placed reliably in the bag. Two radial capsular tears occur at the edges of the capsulotomy, but they are symmetrical, and their positions are known, so the haptics can be placed to avoid them.

Posterior capsule tears were the most frequent complication, occurring in $7.2 \%$ of eyes. Other reports suggest that capsular tears may be more common in Africa than in industrialised countries..$^{2-5}$ This may be because of the type of cataract, as dense, hypermature lenses are more common in Africa. Alternatively, the relative inexperience of the operator, and less than optimal equipment and conditions may also play a part.

Vitreous loss was more common in eyes in which CCC was performed. Given that there was no difference in visual outcome between 
the two methods, is the use of CCC justified? Firstly, the follow up period is short, with an average follow up of just over 1 year. Capsulorhexis would be expected to reduce the frequency of posterior capsule opacity, and lens decentration, both of which are likely to become manifest years after surgery. It may be that the follow up is too short to demonstrate the benefits of CCC. Secondly, the high rate of vitreous loss occurred during the first 100 CCC cases, and is probably the result of the surgeon's learning curve rather than indicating an inherently dangerous technique. These results suggest that, although CCC offers theoretical advantages over a linear capsulotomy, these remain unproved in Africa. The technique is more difficult to learn, and may be impossible in hypermature lenses. For these reasons we advocate linear capsulotomy as the technique of choice when training new surgeons in ECCE and PC-IOL in Africa.

Viscoelastics were used for IOL insertion in almost all cases. Solutions of hydroxypropyl methylcellulose can be purchased in India for less than $£ 3$. Each vial can be used for several cases. Although this does lead to a slightly increased cost per operation, we believe that this added cost is negligible compared with the potential cost of the complications that might occur without viscoelastic. It is quite possible to insert the IOL under air alone. However, we would advocate that viscoelastics should always be available, as it is not always possible to predict which eyes will require the additional protection of a viscoelastic, and which eyes will not.

VISUAL RESULTS

The visual results are similar to those obtained in South Africa, and Asia. ${ }^{3-6}$ Over $90 \%$ of patients obtained a best corrected acuity of $6 / 18$ or better. However, many of these patients required astigmatic correction to achieve their best corrected vision. Very few of our patients can afford the cost of astigmatic lenses. For this reason we believe that the uncorrected visual acuity is likely to correlate more closely with the actual postoperative situation. Nearly three quarters of the eyes had an uncorrected acuity of $6 / 18$ or better at 2 months after surgery. This figure was improved slightly by preoperative IOL power calculation. Since biometry necessitates the purchase of a keratometer, and an A-scan ultrasound machine, as well as requiring a larger inventory of IOLs, it is associated with significant additional costs. If the benefit conferred by preoperative IOL power calculation is marginal, then the provision of biometry equipment for cataract surgeons is not a priority in most situations in Africa.

As uncorrected aphakia may be an important cause of blindness in Africa, ${ }^{78}$ it is encouraging to note that $95.8 \%$ of eyes had an uncorrected acuity of $6 / 36$ or better. Community based studies suggest that, in developing countries, as many as one third of aphakic patients do not wear their glasses. ${ }^{79}$ Since uncorrected aphakic vision is usually worse than $6 / 60$, our patients would appear to have benefited from the use of an IOL.
Good visual acuity at 2 months was strongly predictive of good acuity at 1 year later in the 140 eyes who had paired data at 2 months and 1 year after surgery. As long term follow up is difficult to obtain in Africa, it is helpful to know that the vision at 2 months is a useful guide to the likely level of vision at 1 year or later.

Only $1.5 \%$ of eyes had a poor visual outcome (best corrected acuity <6/60). This contrasts with the reports from west Africa, in which $19-25 \%$ of patients had a vision of less than $6 / 60 .^{2-5}$ Of the six eyes, three had pre-existing retinal conditions. One patient had significant capsular opacity and refused capsulotomy. One patient lost vision from proliferative diabetic retinopathy, and it is possible that the progress of her retinopathy was accelerated by cataract surgery. One eye was lost from a suppurative keratitis which failed to respond to antibiotics or antivirals. It is probable that the postoperative regimen of topical steroids exacerbated his infection. Only three of the six eyes had a poor visual outcome as a result of either the surgery, or postoperative complications. This represents a surgical failure rate of $0.7 \%$.

\section{POSTOPERATIVE COMPLICATIONS}

The most frequent problem was severe uveitis. Uveitis, following intraocular surgery, may be more severe in heavily pigmented eyes. We use topical steroids perioperatively, and for at least 4 weeks following surgery. In some cases repeated subconjunctival injections of steroid, or a short course of systemic steroids may be necessary. The typical fibrinous uveitis may not become apparent until 5-7 days after surgery. We believe that, in Africa, it is important to see all ECCE and PC-IOL patients 1 week after surgery. If the patient cannot return as an outpatient, it may be necessary to keep him or her in hospital.

No cases of endophthalmitis were seen. We attribute this partly to preoperative antimicrobial therapy which includes topical antibiotics during the 24 hours preceding surgery, and povidone iodine $5 \%$ eye drops immediately preoperatively. ${ }^{10}$

Very few eyes developed significant posterior capsule opacification. It has been reported that mature cataracts are at significantly lower risk of capsule opacity. ${ }^{11}$ As many of our patients had mature lenses, this would reduce the likelihood of capsular opacification. All IOLs used had biconvex optics, and every effort was made to place the IOL in the capsular bag. This should also help to prevent capsule opacity. Finally, it should be noted that only $38 \%$ of the patients were followed up for more than 1 year. Capsule opacification may occur several years after surgery. Despite the limitations of these data, it is encouraging to note that the risk of visually significant capsule opacity during the first 2 years after surgery is less than $5 \%$. It is likely that the frequency of capsule opacity will increase with the passage of time, but there is no evidence, in this cohort of patients, that it will become a major cause of blindness. Ruit et al reported a $21.9 \%$ frequency of "visually significant" capsule opacity in their series from 
Nepal. ${ }^{6}$ However, over $99 \%$ of their patients had a corrected acuity of $6 / 60$ or better, so it is safe to assume that the capsule opacity very rarely reduced the vision below $6 / 60$.

BIOMETRY AND REFRACTIVE OUTCOME

Biometry was associated with a significantly lower risk of a large spherical error. However, this did not lead to substantial improvements in uncorrected visual acuity. This may be because the average follow up in the patients who had biometry was shorter. As there is an "against the rule" shift in astigmatism following ECCE and PC-IOL, longer follow up was associated with a reduction in the cylinder required. Longer follow up of the biometry patients might show an improvement in uncorrected visual acuities.

One fifth of eyes had at least 2 dioptres of astigmatism, and would not have been adequately corrected with a spherical lens. Few of our patients can afford the cost of an astigmatic lens. It is, therefore, particularly important to minimise postoperative astigmatism. Although the use of a clear corneal incision might help to reduce the incidence of postoperative fibrinous uveitis, we believe that this is outweighed by the increased astigmatism associated with a corneal section. We used interrupted sutures, however, it is possible that a continuous stitch might have caused less astigmatism in some cases. We believe it is important to refract patients at 2 months, and, if there is significant (at least 4 dioptres) with the rule astigmatism, the sutures should be removed. Lesser degrees of astigmatism will probably reduce without intervention. Against the rule cylinder is likely to be exacerbated by removal of superior limbal sutures.

\section{Conclusion}

This study confirms that it is possible to obtain good results with ECCE and PC-IOL in Africa, even during the transition from routine ICCE to ECCE and PC-IOL. There are few complications from the use of an IOL, and even without biometry, nearly three quarters of the eyes obtained an uncorrected vision of $6 / 18$ or better.
As the population in Africa increases, and gets older, cataract blindness is likely to increase as well. Without a corresponding increase in the number of cataract operations performed, there will be an exponential rise in the numbers of people blinded by cataract. The greatest obstacle to increasing the number of cataract operations in Africa is the reluctance of patients to come for surgery. ${ }^{12}$ This is the result of several different factors including a scarcity of trained eye care providers, the isolation of much of the rural population, the cost of transport and treatment, and the lack of adequate facilities and materials. Another important barrier is the population's attitude to cataract surgery. People may not be aware that cataract is treatable, or, even worse, they may be aware that an operation is possible, but believe that the outcome is so poor that surgery is rarely justified. The improved visual outcome obtained by the use of an IOL should persuade many more patients that cataract surgery is worthwhile.

1 Foster A. Who will operate on Africa's 3 million curably blind people? Lancet 1991;337:1267-9.

2 Cook NJ. Evaluation of high volume extracapsular cataract extraction with posterior chamber lens implantation in Sierra Leone, west Africa. Br F Ophthalmol 1996;80:698701 .

3 Welsh NH. Extracapsular cataract extraction with and without intra-ocular lenses in black patients. S Afr Med F 1992; 81:357-60.

4 Apple DJ, Solomon KD, Tetz MR, et al. Posterior capsule opacification. Surv Ophthalmol 1992;37:73-116.

5 Egbert PR, Buchanan M. Results of extracapsular cataract surgery and intraocular lens implantation in Ghana. Arch surgery and intraocular lens in
Ophthalmol 1991;109:1764-8.

6 Ruit S, Robin AL, Pokhrel RP, et al. Extracapsular cataract extraction in Nepal. 2-year outcome Arch Ophthalmol 1991;109:1761-3.

7 Bucher PJ, Ijsselmuiden CB. Prevalence and causes of blindness in the northern Transvaal. Br F Ophthalmol 1988; 72:721-6.

8 Cook CD, Stulting AA. Impact of a sight-saver clinic on the prevalence of blindness in northern KwaZulu. $S$ Afr Med $\mathcal{F}$ 1995;85:28-9.

9 Hogeweg M, Sapkota YD, Foster A. Acceptability of aphakic correction. Results from Karnali eye camps in Nepal. Acta Ophthalmol 1992;70:407-12.

10 Isenberg SJ, Apt L, Yoshimori R, et al. Outpatient topical use of povidone-iodine in preparing the eye for surgery. Ophthalmology 1989;98:1769-75.

11 Argento C, Nunez E, Wainsztein R. Incidence of postoperative posterior capsular opacification with types of senile cataracts. 7 Cataract Refract Surg 1992;18:586-8.

12 Yorston D. Are IOLs the solution to cataract blindness in Africa? Br f Ophthalmol 1999; in press. 